\title{
Analysis of differentially expressed genes and microRNAs in alcoholic liver disease
}

\author{
YING LIU $^{1 *}$, SHAO-HUA CHEN ${ }^{2 *}, \mathrm{XI} \mathrm{JIN}{ }^{2}$ and YOU-MING $\mathrm{LI}^{2}$ \\ ${ }^{1}$ Department of Gastroenterology, Heilongjiang Province Hospital, Harbin, Heilongjiang 150036; \\ ${ }^{2}$ Department of Gastroenterology, The First Affiliated Hospital, Zhejiang University \\ School of Medicine, Hangzhou, Zhejiang 310003, P.R. China
}

Received September 24, 2012; Accepted November 16, 2012

DOI: 10.3892/ijmm.2013.1243

\begin{abstract}
The purpose of this study was to screen differentially expressed genes and microRNAs in order to find a new target for the accurate diagnosis and effective therapy of alcoholic liver disease (ALD) at the gene and microRNA levels. The total RNA of liver tissues was extracted from four groups of patients, ten subjects each. Microarrays were utilized to detect differentially expressed genes and microRNAs. According to gene values, significance levels and false discovery rate with a random variance model, gene ontology (GO) and the Kyoto Encyclopedia of Genes and Genomes (KEGG) database, node genes and key microRNAs in networks were obtained and analyzed. A total of 878 differentially expressed genes and 26 microRNAs were found. In co-expression genetic networks, node genes modulating the network were Acyl-coenzyme A synthetase-3 (ACSF3), Frizzled-5 (FZD5), LOC727987 and C1orf222. In microRNA-gene networks, the key microRNAs were hsa-miR570, hsa-miR-122, hsa-miR-34b, hsa-miR-29c, hsa-miR-922 and hsa-miR-185, which negatively regulated approximately 79 downstream target genes. In the course of ALD, we found 4 differentially expressed node genes and analyzed ACSF3 and FZD5. ACSF3 was significantly upregulated, and was involved in fatty acid and lipid metabolism and accelerated liver injury. These two genes were involved in fatty acids and lipid metabolism. FZD5 was downregulated and reduced the synthesis of membrane transport protein in the hepatic membrane and the membrane stability, and accelerated the liver cell apoptosis process. Six key microRNAs regulated numerous biological functions such as the immune response, the inflammatory response and glutathione metabolism. This finding provides valuable insight into the diagnosis and treatment of ALD.
\end{abstract}

Correspondence to: Dr You-Ming Li, Department of Gastroenterology, The First Affiliated Hospital, Zhejiang University School of Medicine, Hangzhou, Zhejiang 310003, P.R. China

E-mail: hhd1118@163.com

${ }^{*}$ Contributed equally

Key words: alcoholic liver disease, gene ontology, microarray, microRNA, pathway

\section{Introduction}

Alcoholic liver disease (ALD) consists of a spectrum of diseases, including mild liver injury, alcoholic fatty liver, alcoholic hepatitis, and alcoholic cirrhosis that has the potential of progressing to hepatocellular carcinoma. China has a higher prevalence of viral hepatitis than western countries (1), although it has shown a declining trend year by year (2). Alcohol consumption has resulted in a gradual increase in morbidity and mortality of ALD (3). However, the epidemiology of ALD in China remains vague, with only few reports at the provincial level. Previous investigations showed the incidence of ALD was approximately 4-5\% in Zhejiang, Xi'an, Guizhou and Taiwan (4). Due to numerous complications related to ALD and the heavy economic burden of its treatment, the pathogenesis of ALD has attracted the attention of much research. In several Chinese studies, rat models were used to investigate different genes, signaling pathways and different microRNAs in a certain stage of hepatic injury $(5,6)$. Although marked progress has been made at the molecular level, the detailed differentially expressed pathogenic genes and microRNAs which downregulate these genes remain unknown.

High-throughput gene and microRNA microarray technology has been used to explore gene expression and regulation, transcription in various diseases (7). microRNAs often negatively regulate gene expression at the post-transcriptional level by incompletely binding to target sequences within the 3'-UTR, and generally do not affect the expression of mRNA (8). Therefore, we combined these two technologies to screen differentially expressed genes and microRNAs in ALD and analyzed their biological functions. To our knowledge, this is the first report on these differentially expressed genes and microRNAs in different stages during the course of ALD, using patient liver tissues. The results of our study may aid in exploring the pathological mechanisms of ALD, and may provide new targets for early diagnosis and gene therapy.

\section{Materials and methods}

Patients. Subjects from the Gastroenterology Department and the Outpatient Department of Heilongjiang Provincial Hospital (Harbin, China) were enrolled in the study from December 2010 
Table I. Patient characteristics.

\begin{tabular}{lcccc}
\hline Specimen no. & Age & Gender & Drinking duration (year) & Daily alcohol consumption (g) \\
\hline 1. Hepatitis & 35 & $\mathrm{M}$ & 10 & $150-200$ liquor \\
2. Healthy & 67 & $\mathrm{M}$ & 0 & \\
3. Healthy & 36 & $\mathrm{M}$ & 0 & 250 liquor \\
4. Cirrhosis & 68 & $\mathrm{M}$ & 40 & $150 / 2$ bottles liquor/beer \\
5. Cirrhosis & 55 & $\mathrm{M}$ & 25 & $200-300$ liquor \\
6. Cirrhosis & 53 & $\mathrm{M}$ & 30 & 100 liquor \\
7. Hepatitis & 37 & $\mathrm{M}$ & 17 & 4 bottles beer \\
8. Hepatitis & 40 & $\mathrm{M}$ & 15 & 3 bottles beer \\
9. Alcohol-free liver & 45 & $\mathrm{M}$ & 13 & $80-100$ liquor \\
10. Alcohol-free liver & 56 & $\mathrm{M}$ & 27 & $120 / 2$ bottles liquor/beer \\
11. Healthy & 45 & $\mathrm{M}$ & 0 & 11 \\
12. Alcohol-free liver & 49 & $\mathrm{M}$ & & \\
\hline
\end{tabular}

Table II. Liver functions of ALD patients.

\begin{tabular}{|c|c|c|c|c|c|c|c|}
\hline Specimen no. & AST (IU/l) & AST (IU/l) & GGT (IU/l) & ALP (IU/l) & $\mathrm{TB}(\mathrm{mmol} / \mathrm{l})$ & Albumin (g/l) & PT (\%) \\
\hline 1. Hepatitis & 194 & 70 & 236 & 165 & 26.57 & 32 & 67 \\
\hline 4. Cirrhosis & 278.5 & 124.6 & 556.7 & 198.3 & 64.4 & 25 & 62 \\
\hline 5. Cirrhosis & 179.6 & 112 & 434 & 387.9 & 74.6 & 23 & 57 \\
\hline 6. Cirrhosis & 332.2 & 289.1 & 650.2 & 734.3 & 121.5 & 21 & 47 \\
\hline 7. Hepatitis & 208 & 123 & 765 & 531 & 57 & 38 & 71 \\
\hline 8. Hepatitis & 98.4 & 67.1 & 232.5 & 332.4 & 21.8 & 39 & 76 \\
\hline
\end{tabular}

to January 2011. Total RNA of liver tissues was extracted from four groups of patients, with ten subjects in each group: healthy subjects, drinkers without liver disease, alcoholic hepatitis, and alcoholic cirrhosis (Tables I and II). The diagnostic criteria for ALD were based on the guideline for diagnosis and treatment established by the Division of Fatty Liver and Alcoholic Liver Disease, Chinese Society of Hepatology. The protocols of this study were approved by the institutional review board of the First Affiliated Hospital, Zhejiang University School of Medicine and Heilongjiang Province Hospital. Written informed consent was obtained from all participants.

Extraction and labeling of sample RNA. Total RNA of liver tissues was extracted from liver tissue using Invitrogen TRIzol, and then the RNeasy kit (Qiagen, Shanghai, China) was used for further purification of total RNA. Purified total RNA was then stored at $-80^{\circ} \mathrm{C}$ until use. Agar electrophoresis or the Agilent 2100 Bio Analyzer System (Agilent, Santa Clara, CA, USA) was used to analyze the integrality and quality of total RNA.

A total of $500 \mathrm{ng}$ total RNA was used to synthesize double-stranded complementary DNA (cDNA) in vitro using T7-Oligo(dT) as a primer according to the manual of the Ambion Illumina TotalPrep RNA Amplification kit (Ambion, Austin, TX, USA). Double-stranded cDNA was transcribed and synthesized into complementary RNA (cRNA), which was further amplified and labeled with biotin. Labeled cRNA was purified and stored at $-20^{\circ} \mathrm{C}$.
Quantitative measurement of the probe. The cRNA synthesized in vitro and labeled with biotin was quantitatively measured using a RiboGreen kit (Molecular Probes, Carlsbad, CA, USA).

Microarray hybridization and image scanning. A total of $1.5 \mu \mathrm{g}$ purified and biotin-labeled cRNA was dissolved in $10 \mu \mathrm{l}$ water and was then added into GEX-HYB buffer. The mixture was added to the microarray. Microarray hybridization was carried out at $58^{\circ} \mathrm{C}$ in a hybridization oven. The microarray was incubated with EIBC solution and was washed at a high temperature; it was then rinsed with $100 \%$ ethanol, sealed with E1 buffer, stained with Cy3 dye, and dried. Microarray was scanned to extract the signals following hybridization using a high precision laser confocal scanner (Illumina scanner, $0.8 \mathrm{M})$.

Bioinformatic data processing. An Illumina error model was used for the analysis of differentially expressed genes. $\mathrm{P}<0.05$ was considered to indicate statistically significant differences. The false discovery rate (FDR) was used to evaluate the significance of gene ontology (GO) and pathways. By combining the GO database with the Kyoto Encyclopedia of Genes and Genomes (KEGG) database, functional classifications and important pathways of related differentially expressed genes were analyzed. TargetScan and MicroCosm were used to analyze the differentially expressed microRNAs and their negatively regulated genes. 

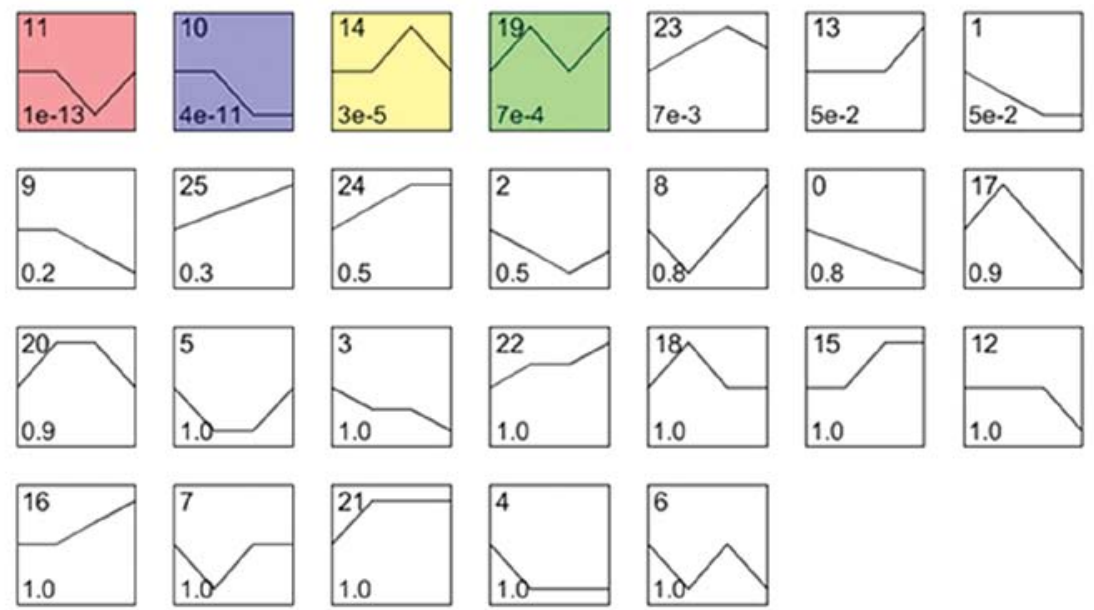

Figure 1. The four significant clustering trends of differentially expressed genes. Trend 11 of the gene expression was invariant at first, then downregulated, and it finally returned to normal level. The variation of trend 14 was contrary to that. Gene expression in trend 10 was invariant at first, then downregulated, then, to a certain extent, it remained unchanged; trend 19 was upregulated at first to the maximum, then downregulated, and when it was downregulated to the healthy level, it was upregulated again.
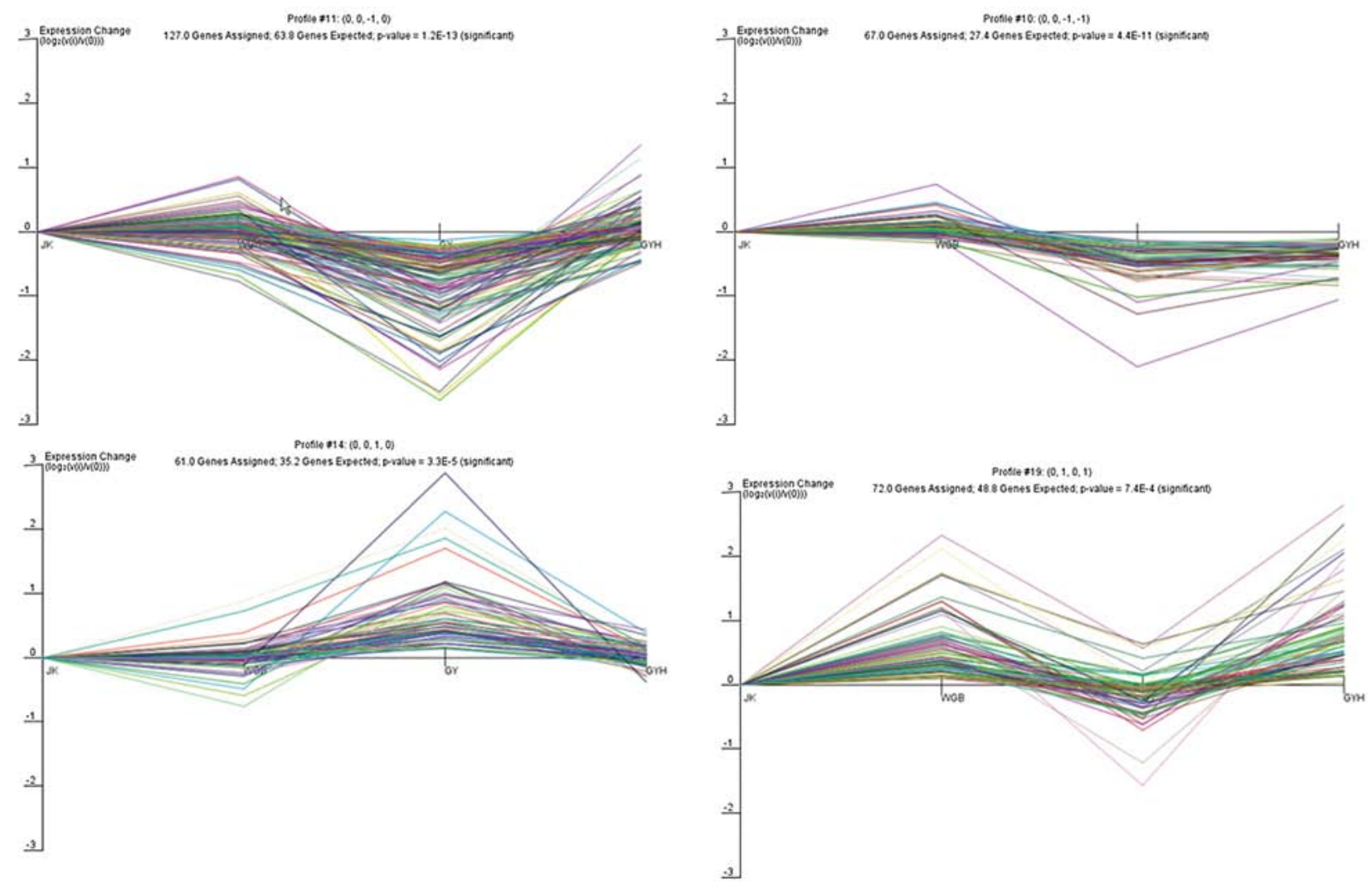

Figure 2. The curve of each significant clustering trend. The horizontal axis represents time (each stage of the disease), vertical axis represents gene expression, the healthy controls as the initial point, the logarithm of ratio of the latter and former as vertical coordinate. A line represents a gene.

\section{Results}

Identification of differentially expressed genes and microRNAs in four groups. In microarray scanning, there were 10 subjects in each group. We chose the random variance model to calculate $\mathrm{P}$-value and FDR of microRNAs. $\mathrm{P}<0.05$ was considered to indicate statistically significant differences. There were 878 differentially expressed genes and 26 microRNAs among the four groups during the stages of ALD.
Clustering trend of differentially expressed genes. Despite the large number of differentially expressed genes obtained by microarray scanning, not all have biological effects on the occurrence and development of ALD. Therefore, we analyzed the gene clustering trend based on the gene expression value. We found the genes with identical expression trends had similar functions or were involved in the same biological processes during ALD. Four significant trends of gene clustering were obtained (Fig. 1). We observed 


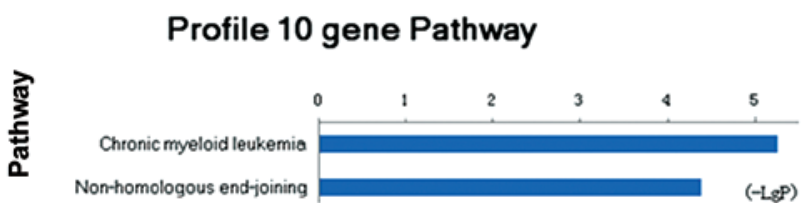

Profile 14 gene Pathway

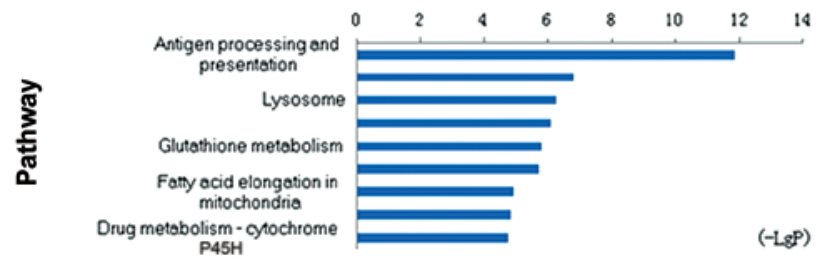

Profile 11 gene Pathway

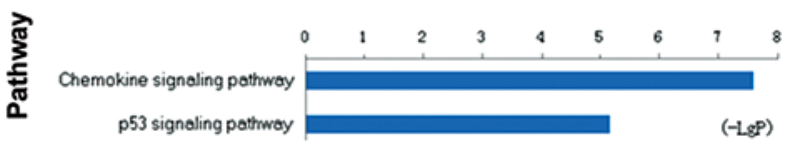

Profile 19 gene Pathway

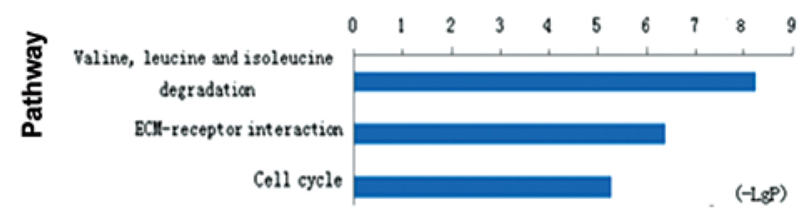

Figure 3. Significant levels of 16 pathways in four clustering trends. Logarithm of P-value of each pathway is the horizontal axis, each pathway name is the vertical axis..

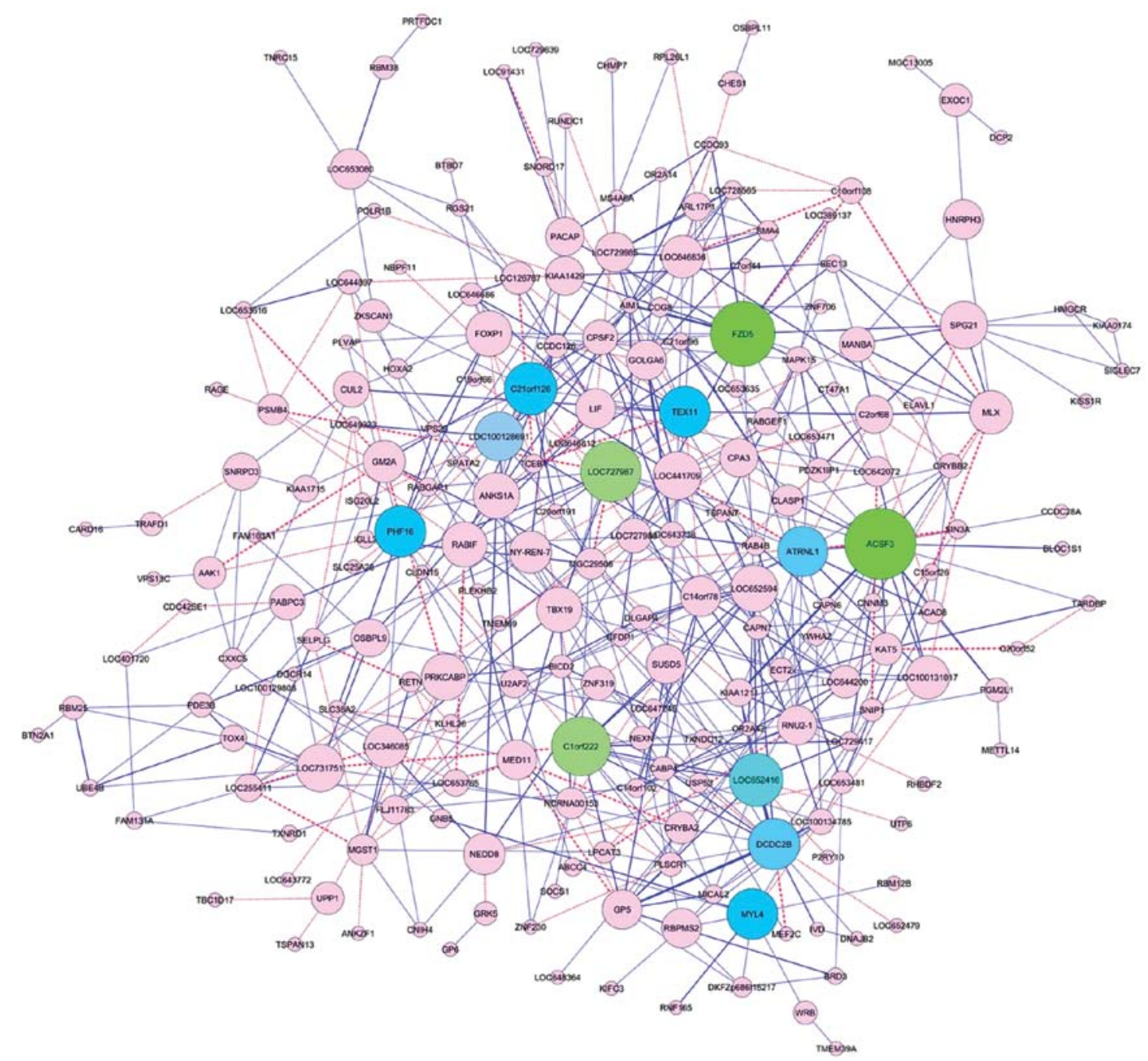

Figure 4. Regulation network of gene expression. The green points represent the node gene, the blue and the pink points represent the first and next stage genes which the node genes regulated, respectively. The area and depth of color of points represent the importance of genes in network. The line represents the interaction among genes, in which the solid line represents gene expressions which are positively correlated (activation), while dashed lines represent the negative correlation (inhibition).

the variation of four trends and found that trend 11 of the gene expression was invariant at first, then downregulated, and it finally returned to normal level, consisting of 127 significantly different genes. The trends 10,14 and 19 had their corresponding variation of gene expression (Fig. 2), consisting of 67, 61, 72 significantly different genes, respec- 


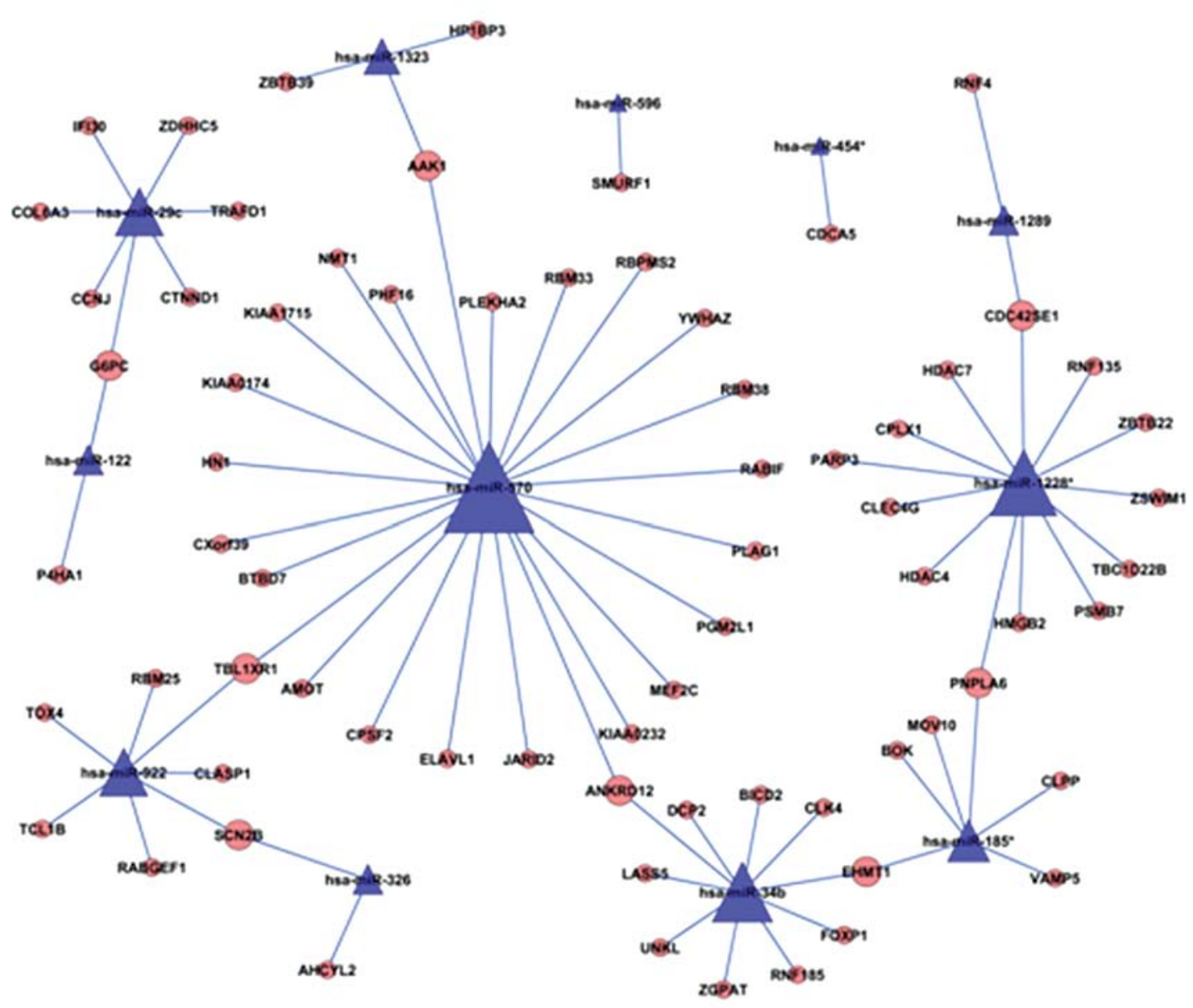

Figure 5. microRNA-gene regulation network. The blue triangles express different microRNAs, the pink circles express genes which were negatively regulated by microRNAs. The area of a triangle is used to determine the important position in the network.

tively. In total, 327 different genes were involved in the four signal clustering trends in ALD.

Significance analysis of gene function and pathway. The genes with identical expression trends had similar functions or participated in the same biological processes during ALD. Therefore, we analyzed functions and pathways of genes in profile clustering trends based on the GO and the KEGG databases. $\mathrm{P}<0.05$ was considered to indicate statistically significant differences. The FDR was used to evaluate the significance of GO and pathway. We took the logarithm of P-value of each GO as the horizontal axis, representing significant level in the trend. The vertical axis represents each corresponding GO-term. In brief, 317 significantly different GOs and their significant level in four clustering trends were described. Sixteen significantly different pathways were found, where 31 corresponding genes participated in these 16 pathways (Table III). Logarithm of P-value of each pathway was the horizontal axis, each pathway name was the vertical axis; the significant level of 16 pathways in four trends is described (Fig. 3).

Construction of the co-expression network of genes. The expression values of genes showing significant trends were used to calculate the co-expression correlation coefficients, which, together with significance level, were used to construct the co-expression network (Table IV and Fig. 4). Betweenness centrality of each gene in the co-expression network was calculated, and describes the degree of importance of the nodes in the whole network. The bigger the betweenness centrality is, the more important the position in the network of genes. The node genes modulating the network were Acyl-coenzyme A synthetase-3 (ACSF3), Frizzled-5 (FZD5), LOC727987 and C1orf222. ACSF3 had the maximal betweenness centrality in the entire gene-modulating network, with a betweenness centrality of 0.102935 , and was downregulated by 21 microRNAs. The second was FZD5, downregulated by 17 microRNAs, whose betweenness centrality was 0.087 . The other two were 0.07683 and 0.073813 , downregulated by 13 and 16 microRNAs, respectively.

Joint analysis of differentially expressed genes and microRNAs. Based on the TargetScan and MicroCosm database, the obtained genes, which had both target regulation and negative expression to microRNAs were used for the microRNA gene regulation network (Fig. 5). The key microRNAs in the gene co-expression network were hsa-miR-570, hsa-miR-122, hsa-miR-34b, hsa-miR-29c, hsa-miR-922, hsa-miR-185, the total number of genes that they negatively regulated was 79 . Hsa-miR-570 was the most important, downregulating 24 genes such as AAK1, TBL1XR1 and ANKRD12. 


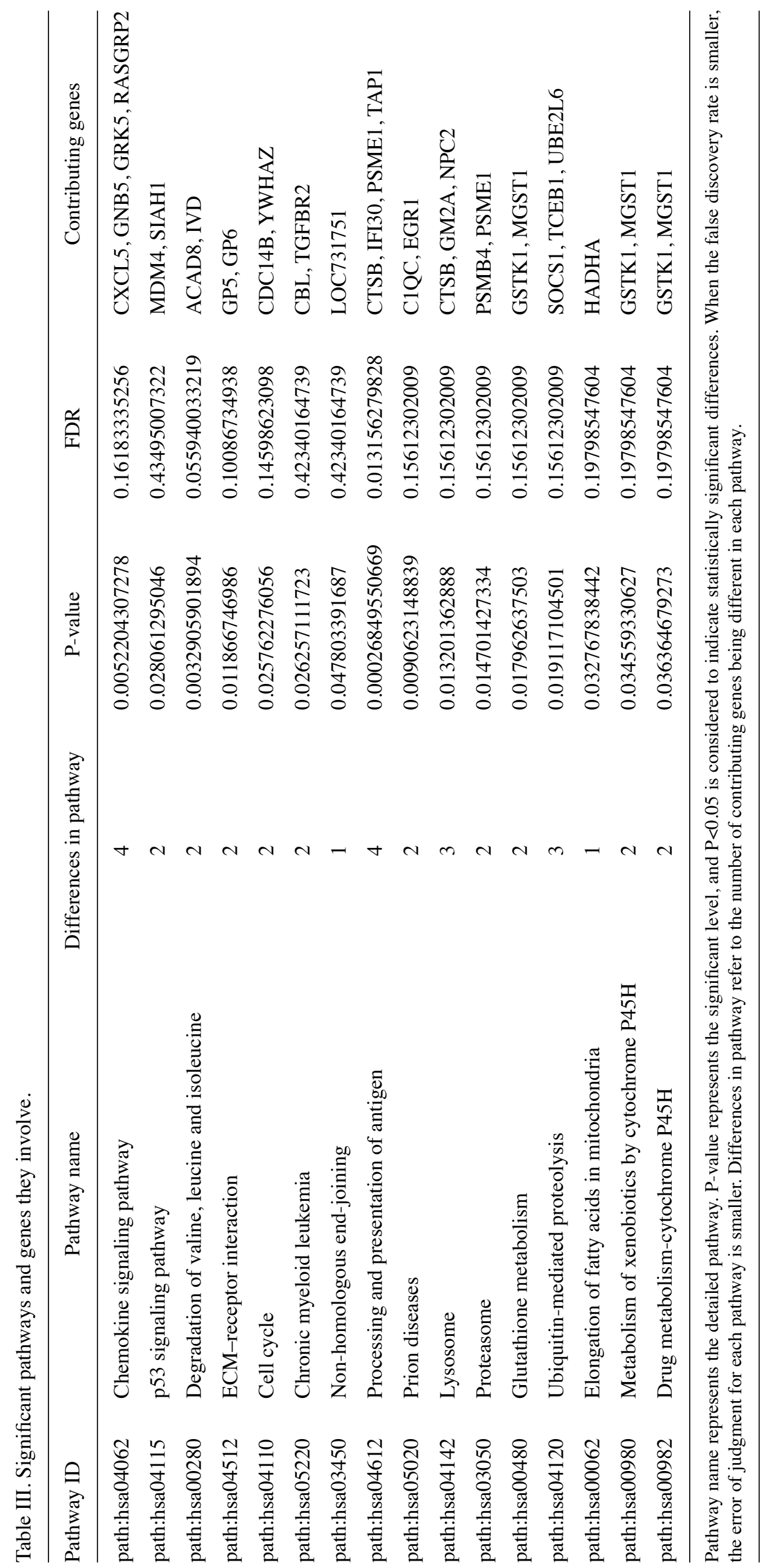




\section{Discussion}

ALD consists of a series of liver diseases induced by long-term and excessive consumption of alcohol. ALD also belongs to the 10 most common causes of mortality in North America and Europe (9). The pathogenesis of ALD has yet to be fully elucidated, although three hypotheses have been postulated (10); the alcohol dehydrogenase (ADH) pathway, microsomal ethanol oxidase system (MEOS), and the catalase (CAT) pathway. Several studies have focused on activated hepatic stellate cell (11), gene polymorphism and mRNA expression in patients (12), lipid peroxidation (LOP) reaction (13), and apoptosis. However, they lacked the detailed mechanism at the genetic function level and microRNA regulation. In addition, most experiments utilized rat models. Therefore, the accuracy and the pathogenesis of ALD remain controversial.

In our study, we employed high work accuracy and efficiency microarray technology, we used hepatic tissue of patients diagnosed with ALD by clinical examination, and we identified 878 differentially expressed genes and 26 microRNAs. This comprised 317 significant GOs, 16 significant pathways and obtained the 5 node genes in the co-expression network and six key microRNAs in the microRNA-gene regulated network.

Acyl-coenzyme A synthetase-3 (ACSF3) was the first key node gene in the gene-modulating network, which was located at 16q24.3 (14). It included nine exons and two transcript variant districts, encoding 576 amino acids (15). ACSF3 binds to thioester and CoA to activate fatty acids for the formation of acyl-coenzyme A, which is involved in the metabolism of fatty acids and lipids as well as ATP binding (16). During the development of ALD, ACSF3 may promote lipid peroxidation, it may interfere with the metabolism of fatty acids (17) and it may aggravate hepatocyte injury and ATP consumption. These effects are unfavorable for hepatocyte repair (18). By calculating gene values, we found that, during dynamic development of ALD, clear upregulation of gene expression of ACSF3 was displayed. Furthermore, the maximal betweenness centrality in the entire gene-modulating network was 0.102935 , indicating its controlling capability and importance. Continuous upregulation of ACSF3 maintains transcriptional activation and, subsequently, strengthens the binding of ACSF3 to thioester and $\mathrm{CoA}$ to activate fatty acids, which results in the irreversible advancement of ALD from hepatitis to cirrhosis.

Frizzled-5 (FZD5) was the second key node gene in the gene-modulating network. It is a multi-transmembrane glycoprotein composed of 585 amino acids. FZD is the receptor of the Wnt protein and has important effects on the Wnt signaling pathway $(19,20)$. If the Wnt signaling pathway is activated, Wnt binds to the FZD receptor and inhibits the degeneration of $\beta$-catenin. The latter is kept at a stable level in the cytoplasm, is gradually transported to the nucleus after accumulation and is related to modulating apoptosis and development $(21,22)$. The gene values of FZD5 in alcoholic hepatitis and cirrhosis were clearly downregulated. The second highest value of betweenness centrality in the entire gene-modulating network was 0.087 . A possible mechanism may be that continuous downregulation of FZD5 during advancement of alcoholic hepatitis to cirrhosis decreased gene activities and reduced the syntheses of multi-transmembrane transport proteins. This would reduce the binding capability of FZD5 with the Wnt protein receptor 
as well as the number and stability of $\beta$-catenin in the cytoplasm. The number of $\beta$-catenin molecules in hepatocyte nucleus and therefore promoted hepatocyte apoptosis (23).

The specific biological functions of the LOC727987 and C1orf222 genes remain unclear and warrant further exploration.

There were six key microRNAs that negatively regulated 79 genes as their downstream targets. Hsa-miR-570 is the most important in the network and controls 24 downstream genes, including Homo sapiens AP2 associated kinase 1 (AAK1). The mu2 subunit of the AP2 complex is known to be phosphorylated in vitro by a copurifying kinase, and it has previously been demonstrated that mu2 phosphorylation is required for transferrin endocytosis (24). Homo sapiens transducin ( $\beta$ )-like 1X-linked receptor 1 (TBL1XR1) is a multifunctional co-repressor of transcription. The structure of this family of molecules is highly conserved and closely related co-repressors have been found in all eukaryotic organisms. Regulation of co-repressor expression and the consequent alterations in transcriptional silencing play an important role in the regulation of differentiation $(25,26)$. However, the detailed functions of AAK1 and TBL1XR1 in ALD remain unknown. These 79 regulated genes play numerous biological functions, including immune response, activity of cancer gene, inflammatory mediated response, apoptosis process, cell cycle, glutathione metabolism, metabolism of xenobiotics by cytochrome $\mathrm{P} 45 \mathrm{H}$, drug metabolism, proteolysis, and fatty acid elongation in mitochondria. Therefore, the downstream genes regulated by microRNAs participate in numerous complex biological mechanisms in the process of ALD. If the activities of node genes and key microRNAs were inhibited, or their regulatory pathways among other genes in the network were inactivated, the whole regulatory network would be paralyzed, and the occurrence and development of ALD would then be influenced.

We previously examined microRNA expression in different stages in nonalcoholic fatty liver using rat models, and gained certain insight (27). However, we need to verify the conclusions and the detailed functions of these node genes and regulatory mechanism of the key microRNAs in a large number of samples. Real-time qPCR was used to confirm the expression of these genes. Although there is some deficiency in our experiment design, data analysis and writing in our study, we believe these data provide a theoretical basis for further studies on the pathogenesis and gene therapy of ALD.

\section{Acknowledgements}

This study was supported by the National Key Technology R\&D Program of China (no. 2008BAI52B03) and the Natural Science Foundation of Heilongjiang Province (no. D201168).

\section{References}

1. Kane M: Global programme for control of hepatits B infection. Vaccine 13 (Suppl 1): S47-S49, 1995.

2. Lu FM and Zhuang H: Management of hepatitis B in China. Chin Med J (Engl) 122: 3-4, 2009.

3. Li YM, Chen WX, Yu CH, et al: An epidemiological survey of alcoholic liver disease in Zhejiang province. Zhonghua Gan Zang Bing Za Zhi 11: 647-649, 2003 (In Chinese).
4. Li YM: Alcoholism and alcoholic liver disease: focusing on epidemiological investigation in Asia. Hepatobiliary Pancreat Dis Int 4: 170-172, 2005.

5. Chen F, Zhu HH, Zhou LF, et al: Genes related to the very early stage of ConA-induced fulminant hepatitis: a gene-chip-based study in a mouse model. BMC Genomics 11: 240, 2010.

6. Guo CJ, Pan Q, Cheng T, Jiang B, Chen GY and Li DG: Changes in microRNAs associated with hepatic stellate cell activation status identify signaling pathways. FEBS J 276: 5163-5176, 2009.

7. Calin GA, Sevignani C, Dumitru CD, et al: Human microRNA genes are frequently located at fragile sites and genomic regions involved in cancers. Proc Natl Acad Sci USA 101: 2999-3004, 2004.

8. Chitwood DH and Timmermans MC: Target mimics modulate miRNAs. Nat Genet 39: 935-936, 2007.

9. Stinson FS, Grant BF and Dufour MC: The critical dimension of ethnicity in liver cirrhosis mortality statistics. Alcohol Clin Exp Res 25: 1181-1187, 2001.

10. Chi BR, Sun Y and Wu Y: The national alcoholic liver disease, multicenter survey analysis. Chung Hua Hsiao Hua Tsa Chih 27: 231-234, 2007 (In Chinese).

11. Ehrig T, Bosron WF and Li TK: Alcohol and aldehyde dehydrogenase. Alcohol Alcohol 25: 105-116, 1990.

12. Lieber CS: Alcohol and the liver: 1994 update. Gastroenterology 106: 1085-1105, 1994.

13. Yang S, Zhu H, Li Y, et al: Mitochondrial adaptations to obesityrelated oxidant stress. Arch Biochem Biophys 378: 259-268, 2000.

14. Sloan JL, Johnston JJ, Manoli I, et al: Exome sequencing identifies ACSF3 as a cause of combined malonic and methylmalonic aciduria. Nat Genet 43: 883-886, 2011.

15. Watkins PA, Maiguel D, Jia Z and Pevsner J: Evidence for 26 distinct acyl-coenzyme A synthetase genes in the human genome. J Lipid Res 48: 2736-2750, 2007.

16. Black PN, Zhang Q, Weimar JD and DiRusso CC: Mutational analysis of a fatty acyl-coenzyme A synthetase signature motif identifies seven amino acid residues that modulate fatty acid substrate specificity. J Biol Chem 272: 4896-4903, 1997.

17. Witkowski A, Thweatt J and Smith S: Mammalian ACSF3 protein is a malonyl-CoA synthetase that supplies the chain extender units for mitochondrial fatty acid synthesis. J Biol Chem 286: 33729-33736, 2011.

18. Hendrickson SL, Lautenberger JA, Chinn LW, et al: Genetic variants in nuclear-encoded mitochondrial genes influence AIDS progression. PLoS One 5: e12862, 2010.

19. Terabayashi T, Funato Y, Fukuda M and Miki H: A coated vesicle-associated kinase of $104 \mathrm{kDa}$ (CVAK104) induces lysosomal degradation of frizzled 5 (Fzd5). J Biol Chem 284: 26716-26724, 2009.

20. Thiele S, Rauner M, Goettsch C, et al: Expression profile of WNT molecules in prostate cancer and its regulation by aminobisphosphonates. J Cell Biochem 112: 1593-1600, 2011.

21. Pereira C, Schaer DJ, Bachli EB, Kurrer MO and Schoedon G: Wnt5A/CaMKII signaling contributes to the inflammatory response of macrophages and is a target for the antiinflammatory action of activated protein $\mathrm{C}$ and interleukin-10. Arterioscler Thromb Vasc Biol 28: 504-510, 2008.

22. Weidler C, Schaumburger J, Miller LE, Scholmerich J and Straub RH: Synovial density of frizzled 5-positive cells does not differ between patients with RA and OA and is independent of inflammation. J Rheumatol 31: 1874-1875, 2004.

23. Tamai K, Zeng X, Liu C, et al: A mechanism for Wnt coreceptor activation. Mol Cell 13: 149-156, 2004.

24. Conner SD and Schmid SL: Identification of an adaptorassociated kinase, AAK1, as a regulator of clathrin-mediated endocytosis. J Cell Biol 156: 921-929, 2002.

25. Yoon HG, Chan DW, Huang ZQ, et al: Purification and functional characterization of the human N-CoR complex: the roles of HDAC3, TBL1 and TBLR1. EMBO J 22: 1336-1346, 2003.

26. Zhang XM, Chang Q, Zeng L, Gu J, Brown S and Basch RS: TBLR1 regulates the expression of nuclear hormone receptor co-repressors. BMC Cell Biol 7: 31, 2006.

27. Jin X, Ye YF, Chen SH, Yu CH, Liu J and Li YM: MicroRNA expression pattern in different stages of nonalcoholic fatty liver disease. Dig Liver Dis 41: 289-297, 2009. 